\title{
Clinical Features and Factors Associated With Surgical Treatment in Patients With Complicated Colonic Diverticulitis
}

\author{
Pill Sun Paik, Jung-A Yun \\ Department of Surgery, Hanyang University Guri Hospital, Hanyang University College of Medicine, Guri, Korea
}

Purpose: Colonic diverticulitis is uncommon in Korea, but the incidence is rapidly increasing nowadays. The clinical features and the factors associated with complications of diverticulitis are important for properly treating the disease.

Methods: A retrospective review of the medical records of 225 patients that were prospectively collected between October 2007 and September 2016 was conducted.

Results: Diverticulitis was detected mainly in men and women aged 30 to 50 years. Diverticulitis more frequently affected the right colon $(\mathrm{n}=194,86.2 \%)$, but age was higher in case of left colonic involvement ( 42 years vs. 57 years, $\mathrm{P}<0.001$ ). Percentages of comorbidities $(65.6 \%$ vs. $23.8 \%, \mathrm{P}<0.001)$, complications $(65.6 \%$ vs. $6.2 \%, \mathrm{P}<0.001)$, and surgical treatment $(50.0 \%$ vs. $4.1 \%, \mathrm{P}<0.001)$ were significantly higher in patients with left colonic diverticulitis. In the multivariate analysis, a risk factor for complicated diverticulitis was left colonic involvement $(\mathrm{P}<0.001$; relative risk [RR], 47.108; 95\% confidence interval [CI], 12.651-175.413). In complicated diverticulitis, age over 50 was the only significant risk factor for surgical treatment $(\mathrm{P}=0.024$; RR, 19.350; 95\% CI, 1.474-254.023).

Conclusion: In patients over 50 years of age with left colonic diverticulitis, a preventive colectomy should be reconsidered as one of the options for treatment.

Keywords: Colonic diverticulitis; Complicated diverticulitis; Clinical characteristics; Risk factor; Surgical treatment

\section{INTRODUCTION}

Diverticulosis is the most common benign colonic disease. In people below 40 years of age, diverticulosis has a prevalence rate of less than $10 \%$, but that rate increases to $30 \%$ for people in the age bracket from 40 to 60 and to $50 \%-70 \%$ for people 80 years of age and older $[1,2]$. Diverticular diseases, such as diverticulitis, abscess, perforation, peritonitis, fistula, obstruction, and hemorrhage [3], develop later in life in approximately $20 \%$ of patients

Received: July 2,2017 - Accepted: September 21, 2017

Correspondence to: Jung-A Yun, M.D.

Department of Surgery, Hanyang University Guri Hospital, Hanyang

University College of Medicine, 153 Gyeongchun-ro, Guri 11923, Korea

Tel: +82-31-560-2045, Fax: +82-31-566-4409

E-mail: ja.yun0713@gmail.com

(C) 2017 The Korean Society of Coloproctology

This is an open-access article distributed under the terms of the Creative Commons Attribution NonCommercial License (http://creativecommons.org/licenses/by-nc/4.0) which permits unrestricted non-

commercial use, distribution, and reproduction in any medium, provided the original work is properly cited. with diverticulosis [4].

Acute colonic diverticulitis is a structural disease of the colon that is common in Western societies [4]. In the United States, in 2004, diverticular disease ranked as the 3rd most common gastrointestinal diagnosis among patients discharged from the hospital. Additionally, diverticular disease was the 4 th most common reason patients were provided with outpatient care [1]. However, an increase in the incidence of colonic diverticulitis in Asia, including South Korea, has been observed. Eating habit changes in Asian populations, as well as reduced physical activity, industrialization, and increasing mean age, are thought to be associated with the increasing incidence of colonic diverticulitis in those populations [3, 5-8].

With the use of computed tomography (CT evaluation), diverticulitis can be categorized as complicated or uncomplicated. Patients with complicated diverticulitis often present with abscesses, fistulas, obstructions, and/or localized or free perforations whereas those with uncomplicated diverticular disease exhibit colonic wall thickening or pericolic inflammatory changes [3]. 
These complications of colonic diverticulitis are considered important because of their clinical and socioeconomic burden. An increase in the prevalence of diverticular disease can lead to more hospital visits [9], and the severity of the disease can affect the admission rates and the lengths of hospital stay [2].

In terms of both mortality and cost, diverticular disease, as well as the complications of the disease, can be a burden to patients who are diagnosed with it [10]. However, not many studies assessing the factors associated with diverticular complications in Asian populations have been published [5], although the characteristics of diverticular disease in Asian societies appear to be different from those in Western societies. For that reason, this study aimed to investigate in the Korean population the clinical characteristics and the factors responsible for the complications of diverticulitis.

\section{METHODS}

From October 2007 to December 2016, 536 patients visited Hanyang University Guri Hospital because of suspicion of acute colonic diverticulitis. Clinical diagnoses were based on symptoms, physical examinations, and blood tests. Most patients underwent $\mathrm{CT}$ or ultrasonography. The $\mathrm{CT}$ scan criteria for the diagnosis of diverticulitis included localized thickening of the colonic wall to $\geq 5 \mathrm{~mm}$ and signs of inflammation of pericolic fat, with or without abscess formation and/or extraluminal air and/or extraluminal contrast [11]. The ultrasound criteria were an inflamed diverticulum with pericolic infiltration (a hypoechoic line surrounding the diverticulum) and increased echogenicity of the adjacent pericolic fat [12]. Of the 536 patients, the diagnoses for 24 patients were not biopsy-proven during surgery, and those for 201 were confirmed by colonoscopic findings. Patients without evidence of di-

Table 1. Patients' characteristics $(n=225)$

\begin{tabular}{lc}
\hline Characteristic & No. of patients \\
\hline Age $(\mathrm{yr})$ & $44 \pm 13(19-81)$ \\
Sex, women : men & $104(46.2): 121(53.8)$ \\
Body mass index $\left(\mathrm{kg} / \mathrm{m}^{2}\right)$ & $24.19 \pm 3.40(16.66-36.71)$ \\
Smoking & $86(38.2)$ \\
Alcohol & $122(54.2)$ \\
Comorbidities & $67(29.8)$ \\
High fever $\left(\geq 38^{\circ} \mathrm{C}\right)$ & $12(5.3)$ \\
Leukocytosis $\left(\mathrm{WBC}>10,000 / \mathrm{mm}^{3}\right)$ & $158(70.2)$ \\
Location, right : left : both & $193(85.8): 31(13.8): 1(0.4)$ \\
No. of previous episodes & $0.2 \pm 0.5(0-2)$ \\
No. of complicated diverticulitis & $33(14.7)$ \\
Treatment, conservative : surgical & $201(89.3): 24(10.7)$ \\
\hline
\end{tabular}

Values are presented as mean \pm standard deviation (range) or number (\%). WBC, white blood cell. verticulitis on the CT scan were excluded from the study.

This study was a retrospective analysis of prospectively collected medical records. This study was approved by the Institutional Review Board of Hanyang University, Guri Hospital (approval number: 2017-08-016-001). Written informed consent was not needed due to the study being retrospective. Conservative management included antibiotic use, fluid resuscitation, and fasting. Food intake was permitted if laboratory findings such as leukocytosis and C-reactive protein had disappeared or the patient's symptoms had improved. After being able to tolerate food, patients were discharged unless their symptoms interfered with daily life. Surgical treatment was necessitated if the patient had complicated diverticulitis or generalized peritonitis. Furthermore, patients who had concerns, even when the condition was diverticulitis without complications, were treated surgically. All patients with surgical treatment underwent a resection of the involved colon.

Continuous variables are reported as means and standard deviations. All data analyses were performed using IBM SPSS ver. 18.0 (IBM Co., Armonk, NY, USA). The significance of the differences between the pairs of groups was tested using the Student $t$-test or Fisher exact test. Variables with P-values $<0.05$ on the univariate analysis were further analyzed using the Cox regression method for a multivariate analysis.

\section{RESULTS}

\section{Clinical characteristics of patients with acute diverticulitis}

The characteristics of the patients with diverticular disease who were recruited for this study are shown in Table 1 . The mean age of the patients was 44 years; $53.8 \%$ of the patients were women, and $38.2 \%$ of the recruited patients were smokers. The most common site for diverticulitis was the right colon. Forty-one patients

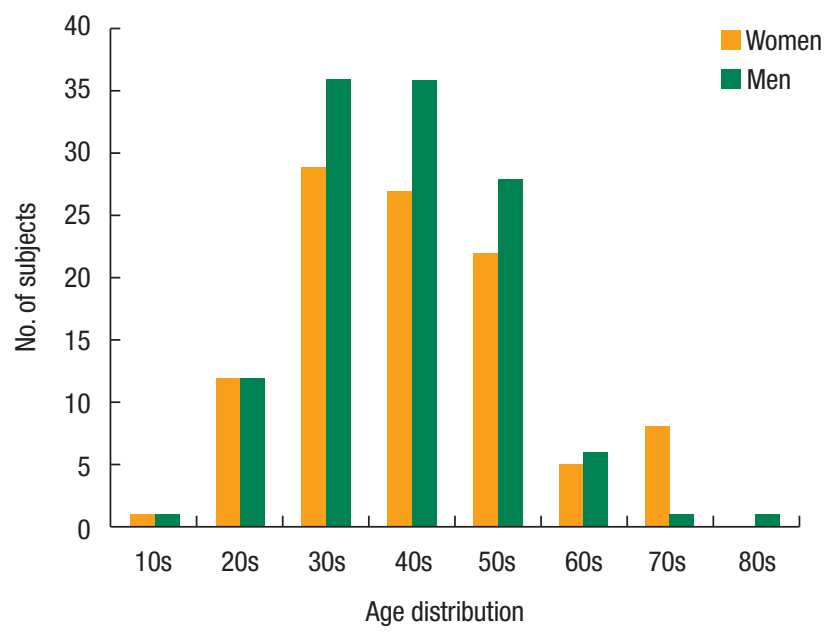

Fig. 1. Kaplan-Meier curve demonstrating the free of surgical recurrence rate as a function of time after perianal surgery for perianal Crohn disease. 
Table 2. Clinical characteristics of patients with and without complicated diverticulitis

\begin{tabular}{lccc}
\hline Characteristic & Without complication $(\mathrm{n}=192)$ & With complication $(\mathrm{n}=33)$ & P-value \\
\hline Sex, women : men & $89(46.4): 103(53.6)$ & $15(45.5): 18(54.5)$ & 0.924 \\
Age $(\mathrm{yr})$ & $42 \pm 12$ & $53 \pm 13$ & $<0.001$ \\
Body mass index $\left(\mathrm{kg} / \mathrm{m}^{2}\right)$ & $24.11 \pm 3.34$ & $24.63 \pm 3.77$ & 0.413 \\
Smoking & $72(37.5)$ & $14(42.4)$ & 0.591 \\
Alcohol & $107(55.7)$ & $15(45.5)$ & 0.274 \\
Comorbidities & $53(27.6)$ & $14(42.4)$ & 0.085 \\
High fever & $8(4.2)$ & $4(12.1)$ & 0.081 \\
Leukocytosis & $128(66.7)$ & $30(90.9)$ & 0.005 \\
Left colon involvement & $11(5.7)$ & $21(63.6)$ & $<0.001$ \\
No. of previous episodes & $0.2 \pm 0.5$ & $0.2 \pm 0.4$ & 0.720 \\
Recurrent diverticulitis & $35(18.2)$ & $6(18.2)$ & 0.995 \\
Surgical treatment & $4(2.1)$ & $20(60.6)$ & $<0.001$ \\
\hline
\end{tabular}

Values are presented as number (\%) or mean \pm standard deviation (range).

Table 3. Multivariate analysis of the risk factors for complicated diverticulitis

\begin{tabular}{lrrc}
\hline Parameter & P-value & RR & $95 \%$ Cl \\
\hline Age, $\geq 50$ yr vs. $<50$ yr & 0.873 & 0.911 & $0.293-2.830$ \\
Comorbidities, yes vs. no & 0.183 & 0.436 & $0.129-1.478$ \\
Left colon involvement, yes vs. no & $<0.001$ & 47.108 & $12.651-175.413$ \\
Recurrent diverticulitis, yes vs. no & 0.632 & 0.741 & $0.217-2.528$ \\
\hline
\end{tabular}

$\mathrm{RR}$, relative risk; $\mathrm{Cl}$, confidence interval.

(18.2\%) were diagnosed with recurrent diverticulitis. Six patients (2.7\%) were admitted with the 3rd attack of diverticulitis. Thirtythree patients (14.7\%) suffered from complications of diverticular disease, among which 20 underwent surgical treatment. Fig. 1 shows the ages of the patients at the onset of acute colonic diverticulitis. Acute diverticulitis was detected mainly among men and women in their 30s and 40s.

Recurrent diverticulitis does not influence the complications or surgical treatment in patients with colonic diverticulitis. Also, 5 out of the 41 patients (12.2\%) with recurrent episodes of diverticulitis underwent surgery, and 19 out of the 184 patients $(10.3 \%)$ who were diagnosed for the first time with diverticulitis had surgical treatment. Except for leukocytosis $(73.4 \%$ vs. $56.1 \%, \mathrm{P}=$ 0.029 ), no statistical differences between patients with nonrecurrent diverticulitis and those with recurrent diverticulitis were noted (Supplementary Table 1).

\section{Clinical characteristics of patients with complicated acute diverticulitis}

In this study, patients who were diagnosed with complicated diverticulitis were older (42 years vs. 53 years, $\mathrm{P}<0.001$ ) and had a higher rate of disease involving the left colon $(5.7 \%$ vs. $63.6 \%, \mathrm{P}<$ 0.001 ). More patients with complicated diverticulitis underwent
Table 4. Clinical characteristics of patients according to left colon involvement

\begin{tabular}{lccr}
\hline Characteristic & $\begin{array}{c}\text { Non-left colon } \\
\text { involvement } \\
(\mathrm{n}=193)\end{array}$ & $\begin{array}{c}\text { Left colon } \\
\text { involvement } \\
(\mathrm{n}=32)\end{array}$ & P-value \\
\hline Sex, women : men & $88(45.6): 105(54.4)$ & $16(50.0): 16(50.0)$ & 0.644 \\
Age $(\mathrm{yr})$ & $42 \pm 11$ & $57 \pm 12$ & $<0.001$ \\
Body mass index $\left(\mathrm{kg} / \mathrm{m}^{2}\right)$ & $24.01 \pm 3.26$ & $25.21 \pm 4.08$ & 0.064 \\
Smoking & $76(39.4)$ & $10(31.3)$ & 0.381 \\
Alcohol & $110(57.0)$ & $12(37.5)$ & 0.040 \\
Comorbidities & $46(23.8)$ & $21(65.6)$ & $<0.001$ \\
High fever & $8(4.1)$ & $4(12.5)$ & 0.073 \\
Leukocytosis & $130(67.4)$ & $28(87.5)$ & 0.021 \\
No. of previous episodes & $0.2 \pm 0.5$ & $0.2 \pm 0.4$ & 0.898 \\
Complication & $12(6.2)$ & $21(65.6)$ & $<0.001$ \\
Surgical treatment & $8(4.1)$ & $16(50.0)$ & $<0.001$ \\
\hline
\end{tabular}

Values are presented as number (\%) or mean \pm standard deviation.

surgery than patients who had diverticulitis without complications $(60.6 \%$ vs. $2.1 \%, \mathrm{P}<0.001)$. Leukocytosis was significantly higher in the patients with complicated diverticulitis $(90.9 \%$ vs. $66.7 \%, \mathrm{P}=0.005$ ) (Table 2). In the multivariate analysis, left colonic diverticulitis was the only significant risk factor for complicated diverticulitis $(\mathrm{P}<0.001$; relative risk [RR], 47.108; 95\% confidence interval [CI], 12.651-175.413) (Table 3).

Differences in clinical characteristics between patients with left-sided diverticulitis and patients with diverticulitis at other locations

Of the 225 patients with diverticulitis, 32 had diverticulitis involv- 
Table 5. Clinical characteristics of patients undergoing surgical treatment for complicated diverticulitis

\begin{tabular}{lccc}
\hline Characteristic & $\begin{array}{c}\text { Conservative } \\
\text { management } \\
(\mathrm{n}=13)\end{array}$ & $\begin{array}{c}\text { Surgical treatment } \\
(\mathrm{n}=20)\end{array}$ & P-value \\
\hline Sex, women : men & $5(38.5): 8(61.5)$ & $10(50.0): 10(50.0)$ & 0.515 \\
Age $(\mathrm{yr})$ & $47 \pm 14$ & $58 \pm 10$ & 0.013 \\
Body mass index $\left(\mathrm{kg} / \mathrm{m}^{2}\right)$ & $24.18 \pm 4.45$ & $24.93 \pm 3.34$ & 0.585 \\
Smoking & $5(38.5)$ & $9(45.0)$ & 0.710 \\
Alcohol & $7(53.8)$ & $8(40.0)$ & 0.435 \\
Comorbidities & $5(38.5)$ & $9(45.0)$ & 0.710 \\
High fever & $2(15.4)$ & $2(10.0)$ & 1.000 \\
Leukocytosis & $11(84.6)$ & $19(95.0)$ & 0.547 \\
Left colon involvement & $6(46.2)$ & $15(75.0)$ & 0.142 \\
No. of previous episodes & $0.3 \pm 0.5$ & $0.1 \pm 0.3$ & 0.183 \\
Recurrent diverticulitis & $4(30.8)$ & $2(10.0)$ & 0.182 \\
\hline
\end{tabular}

Values are presented as number (\%) or mean \pm standard deviation.

ing the left colon, and 193 had diverticulitis at other locations. Patients with left-sided diverticulitis were older ( 42 years vs. 57 years, $\mathrm{P}<0.001$ ). Furthermore, patients with left-sided diverticulitis were less likely to be consumers of alcohol $(37.5 \%$ vs. $57.0 \%$, $\mathrm{P}=0.040)$, had more comorbidities $(65.6 \%$ vs. $23.8 \%, \mathrm{P}<0.001)$, had higher rates of leukocytosis $(87.5 \%$ vs. $67.4 \%, \mathrm{P}=0.021)$, had higher rates of complications ( $65.6 \%$ vs. $6.2 \%, \mathrm{P}<0.001$ ), and underwent more surgical treatments $(50.0 \%$ vs. $4.1 \%, \mathrm{P}<0.001)$ (Table 4).

\section{Clinical characteristics of patients who underwent surgical treatment for complicated diverticulitis}

Of the patients with diverticular complications, 13 were treated conservatively, and 20 were treated surgically. The mean age of the 20 patients who underwent surgery was significantly higher than that of the patients who did not undergo surgery (58 years vs. 47 years, $P=0.013$ ) (Table 5). From the multivariate analysis, age over 50 years was the only risk factor for surgical treatment of diverticulitis with complications $(\mathrm{P}=0.024$; $\mathrm{RR}, 19.350$; 95\% CI, 1.474-254.023) (Table 6).

\section{DISCUSSION}

From our retrospective review, acute diverticulitis was mainly detected in men and women in their 30s and 40s, a relatively young age compared to the ages reported in previous studies [5]. Right colonic diverticulitis occurred more often than left colonic diverticulitis, and complicated diverticulitis was higher in the patients with left colonic diverticulitis. Surgical treatment of complicated diverticulitis was more often performed in older patients over 50 years old, which might have been due to their clinical deteriora-
Table 6. Multivariate analysis of the risk factors for surgical treatment of patients with complicated diverticulitis

\begin{tabular}{lcrl}
\hline Parameters & P-value & RR & \multicolumn{1}{c}{$95 \% \mathrm{Cl}$} \\
\hline Age, $\geq 50$ yr vs. $<50$ yr & 0.024 & 19.350 & $1.474-254.023$ \\
Comorbidities, yes vs. no & 0.243 & 0.268 & $0.029-2.447$ \\
Left colon involvement, yes vs. no & 0.341 & 2.864 & $0.329-24.969$ \\
Recurrent diverticulitis, yes vs. no & 0.196 & 0.218 & $0.022-2.197$ \\
\hline
\end{tabular}

$\mathrm{RR}$, relative risk; $\mathrm{Cl}$, confidence interval.

tion compared with younger patients.

The disease paradigm of diverticulitis, which was previously thought to be a disease of the "old," has changed. The incidence of diverticular disease in younger patients has increased during the last decade $[1,13]$. A study demonstrated that over the recent past, the number of patients younger than 50 years of age with diverticulitis has risen significantly [6], which correlates with the findings of our study. The prevalence of young diverticular patients is thought to be due mainly to changes in dietary habits and lifestyle [6].

Consistent with our study, recent studies showed a right-side preponderance of diverticular diseases in the Asian populations [14]. In contrast to Western populations, diverticular involvement in locations other than the left colon was observed to be more frequent in our study, and a recent study found right-sided diverticulitis to be predominant in Asia, including Korea $[7,15]$ and to be influenced by intraluminal pressure and content [16]. Right colonic diverticula are congenitally-formed true diverticula that are defined as sac-like, entire-bowel-wall herniations whereas left colonic diverticula, usually associated with secondary causes, are false diverticula involving only a mucosal and submucosal protrusion through the colonic muscularis propria [14].

In our study, left colon involvement, which is less common in Asia, was influenced by age and comorbidities. Even though recent publications have reported a dramatic rise in left-sided diverticulitis in younger age groups [13], differently from right-sided diverticulitis, left-sided diverticulitis is well known to depend strongly on age [3], indicating that left-sided diverticulitis is an acquired disease. Changes in dietary habits, increased colonic pressure, defecation habits, and irritable bowel syndrome are some of the secondary causes that are responsible for left-sided diverticular diseases [14].

With increasing age, people tend to have more comorbidities than younger versions of themselves, and in accordance with our study and other recent research, older patients with more comorbidities have greater odds of left-sided diverticulitis, which has an increased tendency of complications. Furthermore, the percentage of patients who underwent surgery was significantly higher for those with left colonic diverticulitis [1]. Right colonic involvement of the disease was more likely to be treated nonsurgically [17]. In our study, a significant age difference was found in the two groups of patients with complicated diverticulitis. In our 
study, for patients with complicated diverticular disease, more patients who were older than 50 years of age underwent surgery while others were treated conservatively.

In the past, operating on patients with recurrent episodes of diverticular disease was an indication $[18,19]$, but in our study, in patients with colonic diverticulitis, recurrent diverticulitis was not a factor that adversely influenced complications or the decision to employ surgical treatment. Also, no evidence of recurrent diverticulitis being more virulent than a single episode of diverticulitis has been reported [20], and elective surgery following recurrent episodes of diverticulitis has not been widely accepted [13]. However, young patients, who can similarly suffer from recurrence and thus require emergent operation as older patients [21], have much more chance than older patients to present with recurred diverticulitis and later need surgical treatment. Even when conservative treatment for complicated diverticulitis with fistula, adhesion or abscess is temporarily successful, long-term resolution of the disease may not be likely [14]. Moreover, recent study suggested that patients who were diagnosed at a young age had increased odds for a colectomy $[15,17]$. Thus, elective surgery for diverticulitis in young patients should be considered because the result of surgical treatment has greatly improved since the advent of laparoscopic surgery and because young patients have a relatively low risk of complications after surgery. Elective surgery may further improve the patient's quality of life in terms of chronic complaints or recurrence of the disease [20].

Our study has several limitations. It was designed retrospectively, and only a small number of patients from a single secondary center in Guri, South Korea, were recruited for our study. The patients who visited our hospital may not have been conservatively treated at primary hospitals; therefore, the severity of the disease may have been worse. In addition, the treatments for the patients recruited for the study were heterogeneous because several methods from a few experts in the center were applied to different patients according to their symptoms and clinical findings. Furthermore, risk factors for diverticulitis, including dietary habits, physical activity, and defecation habits, were not included in our research. Thus, the results of our study cannot be generalized to the entire population.

In conclusion, as long-term diverticular disease in younger patients may lead to complicated diverticulitis because of longer life expectancy, special care should be considered for patients with the disease, even though recurrent diverticulitis does not appear to influence the complications of or the need for surgical treatments in patients with colonic diverticulitis. Also, left colonic diverticulitis may develop complications such as perforations and/ or abscesses, and patients who are older with complications have a tendency of being treated surgically. Therefore, a preventive colectomy should be reconsidered as one of the options for the treatments of patients with left colonic diverticulitis who are older than 50 years of age.

\section{CONFLICT OF INTEREST}

No potential conflict of interest relevant to this article was reported.

\section{SUPPLEMENTARY MATERIAL}

Supplementary Table can be found via http://www.coloproctol. org/src/sm/ac-33-178-s001.pdf.

\section{REFERENCES}

1. Böhm SK. Risk factors for diverticulosis, diverticulitis, diverticular perforation, and bleeding: a plea for more subtle history taking. Viszeralmedizin 2015;31:84-94.

2. Jamal Talabani A, Lydersen S, Ness-Jensen E, Endreseth BH, Edna TH. Risk factors of admission for acute colonic diverticulitis in a population-based cohort study: The North Trondelag Health Study, Norway. World J Gastroenterol 2016;22:10663-72.

3. Chung BH, Ha GW, Lee MR, Kim JH. Management of colonic diverticulitis tailored to location and severity: comparison of the right and the left colon. Ann Coloproctol 2016;32:228-33.

4. Tursi A, Brandimarte G, Di Mario F, Annunziata ML, Bafutto M, Bianco MA, et al. Predictive value of the Diverticular Inflammation and Complication Assessment (DICA) endoscopic classification on the outcome of diverticular disease of the colon: an international study. United European Gastroenterol J 2016;4:604-13.

5. Manabe N, Haruma K, Nakajima A, Yamada M, Maruyama Y, Gushimiyagi M, et al. Characteristics of colonic diverticulitis and factors associated with complications: a Japanese multicenter, retrospective, cross-sectional study. Dis Colon Rectum 2015;58: 1174-81.

6. Jena MD, Marcello PW, Roberts PL, Read TE, Schoetz DJ, Hall JF, et al. Epidemiologic analysis of diverticulitis. Clin Colon Rectal Surg 2016;29:258-63.

7. Barroso AO, Quigley EM. Diverticula and diverticulitis: time for a reappraisal. Gastroenterol Hepatol (N Y) 2015;11:680-8.

8. Strate LL, Keeley BR, Cao Y, Wu K, Giovannucci EL, Chan AT. Western dietary pattern increases, and prudent dietary pattern decreases, risk of incident diverticulitis in a prospective cohort study. Gastroenterology 2017;152:1023-30.e2.

9. Greenwood-Ericksen MB, Havens JM, Ma J, Weissman JS, Schuur JD. Trends in hospital admission and surgical procedures following ED visits for diverticulitis. West J Emerg Med 2016;17:409-17.

10. Cao Y, Strate LL, Keeley BR, Tam I, Wu K, Giovannucci EL, et al. Meat intake and risk of diverticulitis among men. Gut 2017 Jan 9 [Epub]. https://doi.org/10.1136/gutjnl-2016-313082.

11. Ambrosetti P, Jenny A, Becker C, Terrier TF, Morel P. Acute left colonic diverticulitis--compared performance of computed tomography and water-soluble contrast enema: prospective evaluation of 420 patients. Dis Colon Rectum 2000;43:1363-7.

12. Mizuki A, Nagata H, Tatemichi M, Kaneda S, Tsukada N, Ishii H, et al. The out-patient management of patients with acute mild-to- 
moderate colonic diverticulitis. Aliment Pharmacol Ther 2005;21: 889-97.

13. Sartelli M, Catena F, Ansaloni L, Coccolini F, Griffiths EA, AbuZidan FM, et al. WSES Guidelines for the management of acute left sided colonic diverticulitis in the emergency setting. World J Emerg Surg 2016;11:37.

14. Oh HK, Han EC, Ha HK, Choe EK, Moon SH, Ryoo SB, et al. Surgical management of colonic diverticular disease: discrepancy between right- and left-sided diseases. World J Gastroenterol 2014;20:10115-20.

15. Tan KK, Wong J, Yan Z, Chong CS, Liu JZ, Sim R. Colonic diverticulitis in young Asians: a predominantly mild and right-sided disease. ANZ J Surg 2014;84:181-4.

16. Soreide K, Boermeester MA, Humes DJ, Velmahos GC. Acute colonic diverticulitis: modern understanding of pathomechanisms, risk factors, disease burden and severity. Scand J Gastroenterol 2016;51:1416-22.
17. Diamant MJ, Schaffer S, Coward S, Kuenzig ME, Hubbard J, Eksteen B, et al. Smoking is associated with an increased risk for surgery in diverticulitis: a case control study. PLoS One 2016;11: e0153871.

18. Tan JP, Barazanchi AW, Singh PP, Hill AG, Maccormick AD. Predictors of acute diverticulitis severity: a systematic review. Int J Surg 2016;26:43-52.

19. Yoo PS, Garg R, Salamone LF, Floch MH, Rosenthal R, Longo WE. Medical comorbidities predict the need for colectomy for complicated and recurrent diverticulitis. Am J Surg 2008;196:710-4.

20. Andeweg CS, Berg R, Staal JB, ten Broek RP, van Goor H. Patientreported outcomes after conservative or surgical management of recurrent and chronic complaints of diverticulitis: systematic review and meta-analysis. Clin Gastroenterol Hepatol 2016;14:18390.

21. Meyer AA, Sadiq TS. What are the indications for resection after an episode of sigmoid diverticulitis? Adv Surg 2015;49:1-13. 